\title{
A rare complication of chronic subdural hematoma evacuation: brain stem hemorrhage: a case report
}

\author{
Ghassen Gader, Mouna Rkhami, Maher Ben Salem, Mohamed \\ Badri, Kamel Bahri, Ihsèn Zammel
}

Department of Neurosurgery, Trauma and Burns Center, Ben Arous, TUNISIA

\begin{abstract}
Chronic subdural hematoma (CSDH), which commonly affects the elderly, is one of the most frequent, but also benign neurosurgical pathologies. Burr hole drainage is the standard surgical modality for evacuation of a CSDH. This technique is known to be safe, with low morbidity and mortality rates. However, postoperative complications have occasionally been reported. We report the case of a 70-year-old man who presented a fatal brain stem hemorrhage after burr-hole drainage for unilateral chronic subdural hematoma. Asymmetrical and rapid decompression were thought to be leading to vascular disruption or sudden increase in cerebral blood flow, was probably responsible for the secondary brain stem bleeding. Therefore, a slow rate of evacuation of chronic subdural hematomas, as well as rigorous postoperative reanimation, are recommended in order to prevent serious complications.
\end{abstract}

\section{Introduction}

Chronic subdural hematoma is considered among the most benign pathologies to be treated in neurosurgery, with a mortality rate ranging from 0.5 to $4 \%(5,14)$. Burr hole drainage is the most commonly used modality for surgical evacuation of $\mathrm{CSDH}$. This technique is known as safe, easy to perform, with a low morbidity rate ranging from 0 to $9 \%$ (14). However, postoperative complications, including cerebral edema, hematoma recurrence, subdural empyema, tension pneumocephalus, and intracranial haemorrhage in other sites, have been reported with variable frequencies $(13,17)$. Intracranial hematomas occurring as a result of evacuation of haematomas may be located more or less distant from the site of the CSDH. Brainstem bleeding secondary to this surgery is extremely rare, as only 3 cases have been reported in the literature $(1,6)$. We report the case of a 70-year old man who suffered brain stem hemorrhage after unilateral $\mathrm{CSDH}$ evacuation, and discuss the physiopathology of this complication. 


\section{Case presentation}

A 70-year-old man with a history of ischemic stroke of which he kept no sequelae, presented with progressive alteration of the state of consciousness lasting for 5 days. Family reported a minor head trauma following a domestic incident. This trauma occurred 3 weeks before admission. Neurological examination found a comatose patient with a GCS of 8. Pupils were intermediate, reflective and symmetric. Brain CT scan (Figure 1) showed a left frontotemporal subdural hematoma with stigmata of recent bleeding. The heamatoma had a maximum thickness of $1.3 \mathrm{~cm}$ with a midline shift of $0.8 \mathrm{~cm}$. Laboratory studies, including clotting profiles, did not reveal any abnormalities. Emergency evacuation of the hematoma was performed. After burr holes were made, the dural surfaces were exposed and coagulated. The dura and outer membrane on the anterior hole were opened first, followed by the posterior hole a few seconds later later. Opening of the outer membranes allowed dark brown hematoma to gush out via the openings. We tried to evacuate the hematoma gradually, but rapid decompression occurred during the initial period of the procedure due to the high pressure. Thereafter, a subdural drainage system was inserted through the posterior burr hole. The patient's vital signs were stable during the operation, and no elevation of arterial blood pressure was observed. Postoperative, there was no signs of awake. Pupils were in bilateral myosis. Control CT scan made day one postoperative demonstrated regression of the subdural haematoma and of the midline shift, but also detected brain stem hemorrhage that was not present on the preoperative images (Figure 2). The patient remained comtose. He had a respiratory infection on 4 th postoperative day managed with antibiotics. He presented bilateral mydriasis on the 7th day postoperative, and died the day after.
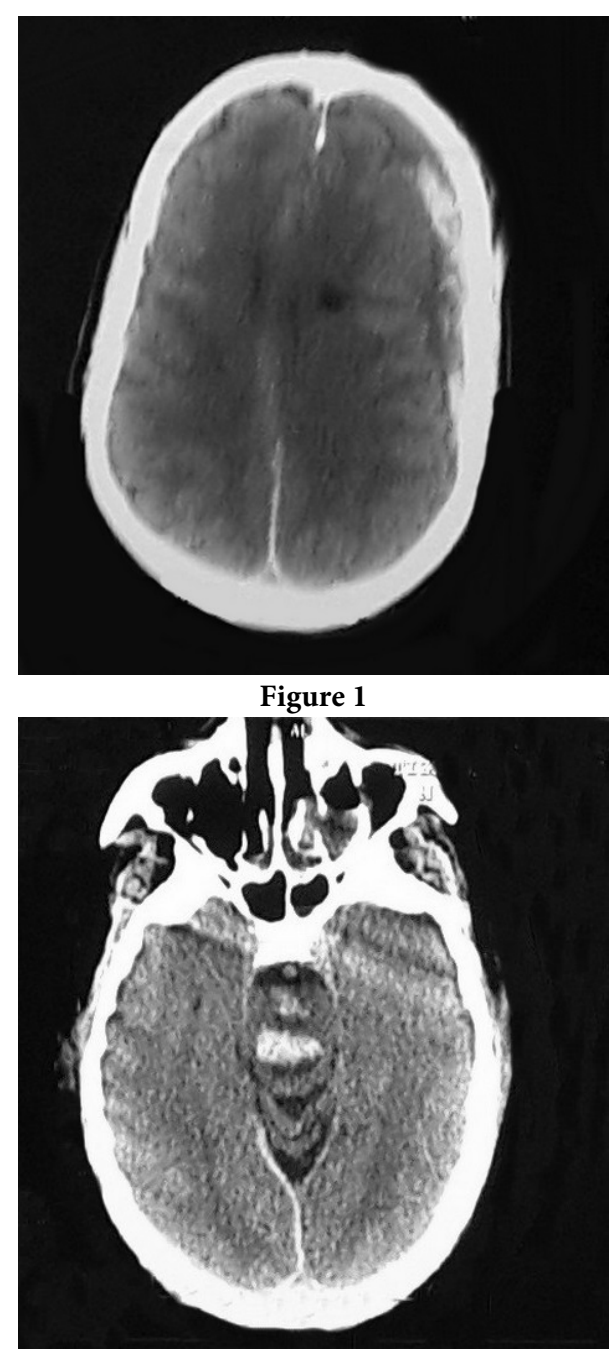

Figure 2 


\section{Discussion}

To the best of our knowledge, only four cases of brain stem hemorrhage as a complication of surgery for CSDH have been reported (8,10-12). Two cases were diagnosed at autopsy, two at postoperative control CT scan. Only one patient survived. Brainstem hematoma was diagnosed in the surviving patient as he presented gait disturbance with mild lower limb weakness. Two of these patients were managed for bilateral chronic subdural hemorrhage, whereas two were operated for a unilateral hematoma. The principal mechanism causing postoperative intracerebral hemorrhage is thought to be a sudden increase in cerebral blood flow combined with defective vascular autoregulation (9). But, the pathophysiology of brain stem hemorrhage following intracranial hematoma decompression is still not clear, since brain stem hemorrhage is often the cause of sudden death, so hemodynamic changes are difficult to study. It is suggested that the brain stem hemorrhage was likely due to damaged vessels caused by increased intracranial pressure (ICP) (4). Rapid alleviation of increased ICP, under conditions of elevated blood pressure, could disrupt the small injured vessels, invariably resulting in brain stem hemorrhage.

In the present case, preoperative $\mathrm{CT}$ showed transtentorial herniation, indicating that the blood vessels near the brain stem might be stretched and distorted (7). The decompression was performed with a short time lag between the opening of the anterior and posterior holes. This decompression was brutal due to the high intracranial pressure, associated to a quick blood draft. Consequently, the vessels surrounding the brain stem may have torn, precipitating the hemorrhage into the brain stem. Vascular disruption could occur easily since the transtentorial herniation was already present, and the vessels surrounding the brain stem had already been maximally stretched. In fact, cranial nerve paresis and brain stem dysfunction have been reported after the removal of CSDHs suggesting that the complications may be due to rapid unilateral decompression, which leads to sudden distortion of the midline structures.

Single photon emission computed tomography has demonstrated that cerebral blood flow in patients with CSDHs is diminished, particularly in the ipsilateral basal ganglia and thalamus (2,3), followed postoperatively by progressive normalization of blood flow. Physiological aging of the cerebral vascular tree is associated with poor tolerance of sudden variations in cerebral blood flow (15). Considering that the present patient was elderly, the increased fragility of the small blood vessels might not have been able to sustain the rapid changes in the brain stem blood flow during decompression of the subdural hematoma. Consequently, the brain stem hemorrhage in the present case was likely due to the mechanical disruption of small fragile vessels, and accentuated by an increase in blood flow caused by breakthrough-like dysregulation triggered by the abrupt supratentorial decompression.

Other factors responsible for brain stem hemorrhage, such as vascular malformations, bleeding tendency, and perioperative hypertension are also evolved (16).

Gradual decompression avoiding rapid changes in blood flow may be accomplished by primary drainage through a frontal burr hole 
and then applying cotton to the burr hole immediately after opening the outer membrane. Continuous closed-system catheter drainage has been proposed for the treatment of $\mathrm{CSDH}$, as it allows the brain to reexpand slowly (10).

\section{Conclusions}

Brainstem hematomas are very rare complications of chronic subdural hematomas evacuation. Slow decompression, control of clotting disorders and maintenance of a correct blood pressure perioperatively may prevent the occurrence of these complications. The present case illustrates that asymmetrical and rapid decompression, which leads to vascular disruption and sudden increase in cerebral blood flow, which may cause brainstem hematoma. Therefore, decompression with a slow rate of evacuation is recommended for the removal of CSDHs, to avoid serious complications such as secondary intracranial hematoma.

\section{Correspondence}

Ghassen Gader

Resident,gastghagad@yahoo.fr,0021698604749

\section{References}

1. Hyam J, Turner J, Peterson D. Cerebellar haemorrhage after repeated burr hole evacuation for chronic subdural haematoma. J Clin Neurosci. 2007;14:83-6.

2. Ishikawa T, Kawamura S, Hadeishi H, Suzuki A, Yasui $\mathrm{N}$, Uemura K. Cerebral blood flow and oxygen metabolism in hemiparetic patients with chronic subdural hematoma. Quantitative evaluation using positron emission tomography. Surg Neurol. 1995;43:130-7.

3. Kim JK, Kim SW, Kim SH. Intracerebral Hemorrhage Following Evacuation of a Chronic Subdural Hematoma. J Korean Neurosurg Soc. 2013;53(2):108.

4. Klintworth G. The pathogenesis of secondary brainstem hemorrhages as studied in an experimental model. Am J Pathol. 1965;47:525-36.

5. Kotwica Z, Brzezinski J. Chronic subdural haematoma treated by burr holes and closed system drainage: personal experience in 131 patients. Br J Neurosurg. 1991;5:461-5.

6. Liang C, Rau C, Lu K, Chen H. Contralateral acute subdural haematoma after burr-hole for chronic subdural haematoma. Injury. 2001;32:499-500.

7. Marupaka S, Sood B. Atypical Duret haemorrhages seen on computed tomography. Emerg Med Australas. 2008;20:180-2

8. McKissock W, Bloom W. Subdural haematoma. A review of 389 cases. Lancet. 1960;1:1365-9.

9. Modesti L, Hodge C, Barnwell M. Intracerebral hematoma after evacuation of chronic extracerebral fluid collections. Neurosurgery. 1982;10:689-93.

10. Park K-J, Kang S-H, Lee H-K, Chung Y-G. Brain Stem Hemorrhage Following Burr Hole Drainage for Chronic Subdural Hematoma. Neurol Med Chir (Tokyo). 2009;49(12):594-597.

11. Patibandla M, Thotakura A, Shukla D, Purohit A, Addagada G, Nukavarapu M. Postoperative hematoma involving brainstem, peduncles, cerebellum, deep subcortical white matter, cerebral hemispheres following chronic subdural hematoma evacuation. Asian J Neurosurg. 2014;0(0):0.

12. Robinson R. Chronic subdural hematoma: surgical management in 133 patients. J Neurosurg. 1984;61:263-8. 13. Rohde V, Graf G, Hassler W. Complications of burrhole craniostomy and closed-system drainage for chronic subdural hematomas: a retrospective analysis of 376 patients. Neurosurg Rev. 2002;25:89-94.

14. Sambasivan M. An overview of chronic subdural hematoma: experience with 2300 cases. Surg Neurol. 1997;47:418-22.

15. Sato M, Nakano M, Asari J, Watanabe K. Intracerebral haemorrhage during surgery for chronic subdural haematoma. J Clin Neurosci. 2007;14:81-3.

16. Sousa J, Golash A, Vaz J, Chaudhary H. Spontaneous intracerebral haemorrhage following evacuation of chronic subdural hematomas. J Clin Neurosci. 2004;11:794-6.

17. Weigl R, Schmiedek P, Krauss J. Outcome of contemporary surgery for chronic subdural haematoma: evidence based review. J Neurol Neurosurg Psychiatry. 2003;74:937-43. 